\title{
Stigmatic Impact of COVID-19 Pandemic on Head \& Neck Cancers Survivors
}

\author{
Atul Kumar Goyal ${ }^{1}$ (i) $\cdot$ Jaimanti Bakshi $^{1} \cdot$ Naresh K. Panda $^{1} \cdot$ Rakesh Kapoor $^{2}$ • \\ Dharam Vir ${ }^{1} \cdot$ Krishan Kumar $^{3} \cdot$ Pankaj Aneja $^{4}$
}

Received: 9 February 2021/Accepted: 12 April 2021/Published online: 22 April 2021

(C) Association of Otolaryngologists of India 2021

\begin{abstract}
Social stigma is the spoilation of the social image of an individual, which leads to the social disapproval of the individual by the community. Patients of many diseases like HIV, deafness, and reproductive disorders often face social disproval. Head \& neck cancer survivors perceived stigma due to the mutilation that occurred after surgical treatment procedures. The novel coronavirus is a recently emerged zoonotic viral agent that affects the respiratory system of humans. Following its
\end{abstract}

Atul Kumar Goyal

atlgyl@gmail.com

Jaimanti Bakshi

drjayabakshi@ymail.com

Naresh K. Panda

npanda59@yahoo.co.in

Rakesh Kapoor

drkapoor.r@gmail.com

Dharam Vir

vir486@gmail.com

Krishan Kumar

keshusony@ rediffmail.com

Pankaj Aneja

drpankajaneja@rediffmail.com

1 Department of Otolaryngology and Head and Neck Surgery (ENT), Post Graduate Institute of Medical Education and Research (PGIMER), Chandigarh 160012, India

2 Homi Bhabha Cancer Hospital and Research Center, Punjab 140901, India

3 Department of Psychiatry, Post Graduate Institute of Medical Education and Research (PGIMER), Chandigarh 160012, India

4 Post Graduate Institute of Medical Education and Research (PGIMER), Chandigarh 160012, India outbreak from the Wuhan city of China, the COVID-19 spreaded fiercely around the globe, forming a pandemic. Since COVID-19 is a contagious disease with no available treatment, social distancing is considered as the best strategy to prevent the geometric spread of infection. With the social distancing model, the head \& neck cancer survivors along with the various other stakeholders perceived stigma being a high-risk group for COVID-19 infection. As the pool of caregivers is diminished due to pandemic, head $\&$ neck cancer survivors face increased social isolation and perceived stigma in asking for help from relatives. At the time of the pandemic, social support is critical to fighting against the disease. Social distancing should be maintained along with communication with the patients through calls, text, and online social platforms. It is not wise to stigmatize disease as, in that case, patients who are infected with the disease will try to hide it and avoid seeking medical care. With the promotion of social distancing, it is crucial to convey awareness regarding not to stigmatize the disease.

Keywords Stigma · COVID-19 · Coronavirus ·

Quarantine $\cdot$ Lockdown $\cdot$ Cancer

\section{Background}

Social stigma is a psychological mindset of society in which a particular person or a group of persons is isolated from the social environment of the community [10]. Social stigma acts as an important factor in determining the psychological health of a patient and has been known to directly influence the outcome of the clinical treatment. Various diseases including HIV, epilepsy, reproductive disorders, deafness have associated social stigma [12]. History has witnessed that various diseases that have 
emerged for the first time such as plague, Ebola and SARS, carry the stigma with them $[7,15]$. The stigma is associated with newly emerged diseases primarily because of fear in the people due to the unknown nature of the disease and lack of treatment [8]. Stigmatization of disease multiplies the adverse consequences as it enhances the suffering of patients and demotivates them for acquiring health care which makes it very difficult to control the spread of disease. Various stakeholders who are working in close proximity to patients also become stigmatized. Apart from causing psychological strain, stigmatization also reported causing considerable economic losses [7]. The novel coronavirus is a zoonotic disease that has recently outbreaks from China and form a pandemic within few months across the globe. Due to the contagious nature of the disease and lack of treatment, a social distancing model has been adopted by the various countries to contain the expansion of infection [18]. With this, various stakeholders and high-risk groups of COVID-19 have started perceiving a social stigma. Head \& neck cancer survivors are one of the high-risk groups who perceived the potential social isolation and stigma in the COVID-19 pandemic [4]. In the present article, we review the stigma associated with the COVID-19 pandemic with special reference to the head \& neck cancers survivors and suggest future studies that could be carried out to manage the stigma at the time of pandemics.

\section{Novel Coronavirus COVID-19 Pandemic}

The novel coronavirus is a disease of the respiratory system, which is caused by the severe acute respiratory syndrome coronavirus 2 (SARS-CoV-2). In February 2020, the world health organization declared the name COVID-19 to coronavirus infection [2]. The outbreak of novel coronavirus occurred from the Wuhan city of China in December 2019 by the zoonotic transmission of viruses from animals to humans. COVID-19 is the fourth emerged novel coronavirus after HCoV-229E, SARS and MERS-CoV [5]. Being contagious, the disease is spreading fiercely around the world within weeks taking the form of a pandemic. The infected person shows the symptoms of fever, fatigue, cough, chest congestion, difficulty in breathing, and inflammation in the lungs [6]. The appearance of symptoms may take a week after the infection, which makes it very difficult to diagnose the infection based on symptoms. Molecular testing methods which confirm the three viral genes- $E, R d R p$ and $N$, in nasopharyngeal and oropharyngeal swabs using the RT-PCR can diagnose disease before the appearance of symptoms [5], but due to high cost and requirement of sophisticated laboratory facilities, mass testing is not feasible at the time of an outbreak. Due to unavailability of cheap detection method and specific pharmaceutical treatment or vaccine of the viral infection, alternative strategies which could limit the infection, is implemented all around the world.

\section{Social Distancing and lockdown in COVID-19}

As COVID-19 is a contagious disease which tends to spread by physical contact from an infected person; proper washing of hands, avoid touching mouth, nose or eyes; covering of mouth and nose with the mask; regular cleaning and disinfection of surfaces having frequent touches; avoid greeting by hands shaking or kissing; reduce exposure to infected patients and large crowds were recommended to contained the spread of COVID-19 [6]. Apart from those measures, social distancing model is prescribed as the most successful strategy to control the spread of the infection. Under social distancing norms, it is recommended to maintain a physical distance of at least up to 6 feet from an infected person [18]. Since there is a large incubation period of up to one week in the appearance of symptoms after infection, it is not possible to know whether a person is infected or not. Although testing of coronavirus can confirm the infection long before the appearance of symptoms, due to lack of cost-effective testing method mass population cannot be tested. Under this scenario, government health agencies suggest maintaining a physical distance to all individuals whether they are infected or not. When the law and legislation enforce social distancing, it created a 'lockdown' situation in many countries [13]. Various recommendations under lockdown include working remotely, shutting down public transportation, staying home, closing of educational institutes, a statutory ban on public meetings and social gatherings, closing of shopping malls, big markets and cinema theaters. Lockdown model help in breaking the geometric spread of infection. Besides this, lockdown is vital to decreasing the load on the healthcare system when no treatment of disease is available, as a potential rise in the number of infected patients could overwhelm the capacity of the healthcare support system [18].

\section{Social Stigma in COVID-19}

Although social distancing looks like a great strategy to contained the spread of infection, it leads to the origin of social stigma because of confusion, anxiety, and fear among the public due to the unknown nature of the disease. Social stigma and discrimination have provoked against the individuals who have a perceived link to disease and lives in a community that is specifically at risk for that particular disease [17]. Various stakeholders including the medical professionals, laboratory technicians, research fellows, police, sanitary workers who work in the environment 
having COVID-19 patients, reported to perceived discrimination in the society [8]. Individuals who remain in close proximity of stakeholders such as caregivers and family members, also get stigmatized as the community started to think that they were more likely to be infected [16]. Individuals who have a recent history of travel to foreign countries and patients affected by the coronavirus perceived the highest discrimination from local neighbors, friends, and even immediate relatives. Even the patients who have successfully recovered from the disease and release from quarantine perceived stigma in society even though they are no longer contagious or at risk of spreading the virus [17]. Stigma creates a feeling of hate, mistrust, anger and fear in the community towards stigmatized individuals. Stigmatized individuals suffer from social rejection, denials of employments and physical violence. Stigma can break social cohesion and enhance social isolation of stakeholders which otherwise might help in the management of pandemic. Without support of various stakeholders, more severe health crises and uncontrolled disease outbreak can be expected at the time of pandemic. The stigma associated with the disease can drive patients to hide the disease which will prevent patients from acquiring medical care. Stigma can drive patients away from screening, testing and quarantine, which in turn results in the spread of infection.

\section{Stigma in Head and Neck Cancer due to COVID-19}

Head \& neck cancers survivors perceived stigma already due to mutilation occurred during the surgical procedures of the treatment phase. Patients feel shame while going out in public places and social functions such as marriage, party, carnivals, or religious function due to facial disfigurements and surgical scars [10]. Most of the time, patients like to stay at home or confine themselves within local surroundings. Due to this social isolation patient feels the anxiety, stress and mood swings which impair a patient's quality of life and reduced overall survival [11]. Head \& neck cancers survivors require regular clinical follow up as cancer surveillance based upon the physical examination of the affected area $[1,3]$. Due to the COVID-19 pandemic, the appointments of patients is postponed to an unknown time frame for rescheduling. Delay in follow-ups and suspicion of recurrence has led to increased anxiety and decreased quality of life of patients [4]. As coronavirus is more likely to infect the patients with a weak immune system, patients with head \& neck cancer, are considered as a high-risk group for coronavirus infection [14]. Moreover, the patients who are currently undergoing radiotherapy or chemotherapy tend to have a weak immune system, represent a high-risk group for COVID-19 infection. Being a high-risk group, head \& neck cancer survivors perceived social resistance from the society which imparts a sense of stigma in cancer patients. Lockdown situation in the country has caused the loss of employment to the millions of people [4]. Both cancer survivors and their caregivers perceived the trouble because of diminished income sources, decreased social contact and support, and enhanced anxiety and depression. Caregivers are the crucial stakeholders in providing support to the head \& neck cancer survivors with the feeding, communication and adherence to the treatment. As the pool of caregivers is diminished due to the COVID-19 pandemic, the head \& neck cancer survivors perceived increased social isolation. Cancer survivors perceived stigma in asking for help from relatives, as being a high-risk group for COVID-19, relatives might think that cancer patients are likely to be COVID-19 positive and it's better to stay away from them [9]. Head and neck cancer survivors, who already perceived enhanced levels of isolation and depression due to their treatment outcomes (eg, facial disfigurement and surgical scars), now face even higher rate of depression [4]. Patients with larynx cancer usually have a consistent residual cough which is symptom of COVID-19 infection. The cancer patients might be considered as COVID-19 positive due to matching symptoms as people who do not have the disease but possess matching characteristics with disease group may also suffer from stigma [8]. Even after the pandemic is contained, the stigma and discrimination could persist in the long term. Therefore, it is crucial to build a support system for the head \& neck cancer survivors which can help patients in overcoming from the stigma associated with the disease.

\section{Recommendations and Future Scope}

Living with a stigmatized chronic disease in the times of pandemic could be a great challenge for patients. A survey that could highlight the barriers that cancer patients faced during the COVID-19 pandemic in getting health support could help in devising strategies for future pandemics. Being a high-risk group, cancer survivors self-isolate them to prevent COVID-19, and therefore it is necessary to assess the effects of enforced social isolation in patients who already reported the stigma. It also might be possible that head \& neck cancer survivors are more likely to perceive isolation because of a history of stigma. Health outcomes after enhanced social isolation also to be evaluated with the likelihood of tobacco chewing, smoking, and alcohol drinking. Understanding of impacts of social isolation on head \& neck cancer survivors, how they are expecting support and in what context they need to be supported, is also required. Short-term interventions that could enhance the social connectedness during the time of isolation also need to be identified. It would be interesting 
to find out how does the COVID-19 impacted adherence to follow-ups, healthcare engagement, and psychological health and rates of perceived stigma among head \& neck cancer survivors. Evaluation of the role of telemedicine for head \& neck cancer care services in enhancing the patient's support and reduction of stigma and discrimination could also be useful. A temporal study that highlights the perception of stigma before and after the COVID-19 pandemic could also be helpful. Preliminary insights regarding the effects of COVID-19 pandemic on the beliefs and attitudes of head \& neck cancer patients about healthcare needs can help in devising effective healthcare intervention. A reliable and straightforward self-reported measure that could measure the discrimination and stigma in pandemics like COVID-19 in cancer patients must be developed for quantitative analysis of the psychological impact of the disease.

\section{Conclusion}

When an outbreak happens, the viruses infect all humans regardless of their sociodemographic profile. Social distancing, quarantine, lockdown is meant to contain the spread of the disease. Patients of head \& neck cancer already suffer from the stigma associated with the mutilation occurring during surgical treatment, it is essential to provide support to those patients at the time of pandemic when social isolation reaches to the extent like never before. Social distancing can be maintained along with communication with the patients through calls, text, and online social platforms. It is not wise to stigmatize COVID19 as, in that case, patients who are infected with the disease will try to hide it due to stigma and will spread the disease to other healthy individuals. With the promotion of social distancing, it is crucial to convey awareness regarding not to stigmatize the disease. It is crucial to build belief in health services, to have empathy with affected patients, and to adopt prescribed law enforcement to safeguard self and others. A social environment, in which the open discussion regarding the disease and its impact can be carried out honestly and effectively, needs to be created. Telemedicine could be a crucial support system for the head \& neck cancer patients who are seeking medical advice during the COVID-19 pandemic.

Acknowledgments Authors highly acknowledge the colleagues who reviewed the present work. Manuscript is proofread by the doc navigator@ Chandigarh.

Authors contribution All authors are equally contributed to present work.
Funding The work in this research is also supported by the Junior Research Fellowship (JRF) provided by ICMR, New Delhi wide Award no. 3/13/JRF -2015/HRD.

\section{Declarations}

Conflict of interest The authors declare that the research was conducted in the absence of any commercial or financial relationships that could be construed as a potential conflict of interest.

Ethical approval The present study has been approved by the institute ethics committee (Letter No. INT/IEC/2019/002539 with Ref No. NK/5657/PhD).

\section{References}

1. Anand A, Goyal AK, Bakshi J, Sharma K, Vir D, Didi A (2018) Yoga as an integrative approach for prevention and treatment of oral cancer. Int J Yoga 11(3):177

2. Anon (2020) Stop the coronavirus stigma now. Nature 580:165

3. Bakshi J, Goyal AK, Singh V, Sannigrahi M, Khullar M (2020) Stage-specific expression analysis ofMMP-2 \& MMP-9 in laryngeal carcinoma. J Cancer Res Ther 16(2):1-4

4. Boakye EA, Jenkins W, Sharma A (2020) Disproportionate impact of COVID-19 pandemic on head and neck cancer survivors. Head Neck 42(6):1329

5. Chatterjee P, Nagi N, Agarwal A, Das B, Banerjee S, Sarkar S et al (2020) The 2019 novel coronavirus disease (COVID-19) pandemic: a review of the current evidence. Indian J Med Res 151(2): 147

6. De Felice F, Polimeni A, Valentini V (2020) The impact of Coronavirus (COVID-19) on head and neck cancer patients' care. Radiother Oncol 147:84

7. Des Jarlais DC, Galea S, Tracy M, Tross S, Vlahov D (2006) Stigmatization of newly emerging infectious diseases: AIDS and SARS. Am J Public Health 96(3):561-567

8. Fayziev SF (2020) The problem of social stigma during a pandemic caused by COVID-19. Int J Adv Sci Technol 29(7):660-664

9. Goyal AK, Bakshi J (2020) Stigmatic Impact of Social Distancing on Head \& Neck Cancer Patients during COVID-19 Pandemic. SPER e-Poster

10. Goyal AK, Bakshi J, Panda NK, Kapoor R, Vir D, Kumar K et al (2020) A hybrid method for the cross-cultural adaptation of selfreport measures. Int J Appl Posit Psychol 6(1):45-54

11. Goyal AK, Bakshi J, Panda NK, Kapoor R, Vir D, Kumar K et al. (2020b) Shame and Stigma Over Long Term Survival in Head and Neck Cancer. CONICA-2020. Panjab University, Chandigarh

12. Goyal AK, Saini J, Chopra T, Panghal S, Dahiya J, Gupta V (2019) Social sports events as an integrative approach to attain the projected impact of HIV/AIDS awareness programs on the local community. AIDSCON-9. IMAComplex, Sec-35, Chandigarh, India

13. Lancet $T$ (2020) India under COVID-19 lockdown. Lancet (Lond, Engl) 395(10233):1315

14. Liang W, Guan W, Chen R, Wang W, Li J, Xu K et al (2020) Cancer patients in SARS-CoV-2 infection: a nationwide analysis in China. Lancet Oncol 21(3):335-337

15. Lin C-Y (2020) Social reaction toward the 2019 novel coronavirus (COVID-19). Soc Health Behav 3(1):1 
16. Ramaci T, Barattucci M, Ledda C, Rapisarda V (2020) Social Stigma during COVID-19 and its impact on HCWs outcomes. Sustainability 12(9):3834

17. Sathyanath S, Sathyanath S (2020) Stigma reduction and provision of mental health services in the public health response to COVID 19. Indian J Comm Health 32:269-272
18. Sen-Crowe B, McKenney M, Elkbuli A (2020) Social distancing during the COVID-19 pandemic: Staying home save lives. Am J Emerg Med 38(7):1519-1520

Publisher's Note Springer Nature remains neutral with regard to jurisdictional claims in published maps and institutional affiliations. 